\title{
Sarna sarcóptica: comunicación de un brote en un grupo familiar y su mascota
}

\author{
José L. Gallegos, Isolda Budnik, Anamaría Peña, Marilena Canales, Mónica Concha y Javier López
}

\section{Sarcoptic mange: report of an outbreak in a family and their pet}

Scabies caused by the genus Sarcoptes scabiei var canis is a prevalent infection in dogs and affects abandoned, malnourished and overcrowded animals, causing hair loss and an intensely pruritic crusting dermatitis. In humans the manifestation is a self-limiting pruritic dermatitis, but persistent cases are described. An outbreak of sarcoptic mange is reported in a family group (seven people, including a 5 month infant and his mother). The infective source was their own house dog who was taken from the street. The diagnosis was confirmed by the detection of mites and eggs in the acarotest of the dog and mites of S. scabei in the infant. Sarcoptic mange should be suspected in individuals with allergic dermatitis who have contact with dogs. Treatment in humans is usually symptomatic and may need miticides if the infection persists. The control of the disease requires an appropriate pet treatment.

Key words: Sarcoptic mange, scabies, zoonoses.

Palabras clave: Sarna sarcóptica, sarna, zoonosis.

\section{Introducción}

$\mathrm{L}$ a sarna sarcóptica es una infección zoonótica de la piel causada por un ácaro de la familia Sarcoptidiae. Pertenece al género Sarcoptes del cual se ha descrito variedades de acuerdo al hospedero (Sarcoptes scabiei var canis, S. scabiei var bovis, S. scabiei var suis, S. scabiei var equi, S. scabiei var aucheniae, S. scabiei var cuniculi, S. scabiei var ovis, S. scabiei var caprae, según si parasitan a perros, bovinos, cerdos, caballos, llamas y alpacas, conejos o cabras; respectivamente). Aunque existe cierto grado de especificidad, puede haber infestaciones cruzadas entre las especies animales dando origen a la condición de hospederos inhabituales dentro de los cuales se encuentra el hombre. La subespecie que infecta al humano (Sarcoptes scabiei var hominis) es distinta a la que afecta a los animales. A veces, el hombre puede contraer la sarna a partir de un animal, pero es infrecuente que cause infestaciones extensas, ya que estos ácaros no suelen reproducirse en humanos y sólo viven algunos días. Esto hace poco probable la transmisión entre humanos ${ }^{1,2}$. Sin embargo, por su alto potencial zoonótico puede afectar a personas que han tenido un contacto prolongado $\mathrm{y}$ estrecho con el animal ${ }^{3,4}$.

Las hembras adultas excavan en la epidermis y depositan sus huevos y heces en madrigueras, lo que da origen a pápulas eritematosas intensamente pruriginosas como resultado de una reacción de hipersensibilidad retardada tipo IV al ácaro, sus fecas y huevos. Las lesiones suelen confundirse con una dermatitis alérgica ${ }^{5-7}$.
En nuestro país, la sarna producida por el género Sarcoptes es la infección parasitaria zoonótica más prevalente en perros de acuerdo a informes de un sistema de vigilancia de mascotas en la Región Metropolitana ${ }^{8,9}$.

En los perros afecta preferentemente a cachorros y animales senescentes, generalmente abandonados, desnutridos y que viven en condiciones de hacinamiento. En ellos causa una dermatitis costrosa intensamente pruriginosa, amplias zonas de pérdida de pelo, descamación, inflamación y sobreinfección bacteriana. Los gatos rara vez se infestan y cuando ocurre se debe generalmente a que son portadores de inmunodeficiencia felina.

Reportamos un brote de sarna sarcóptica en un grupo familiar cuya fuente de infección fue su mascota canina.

\section{Caso clínico}

Se recibió en el Hospital Veterinario Puente Alto a un paciente canino, macho, mestizo, de tres meses de edad (cachorro), con antecedente de haber sido recogido desde la calle un mes antes. El motivo de consulta fue un intenso prurito manifestado como rasquido por lo que se encontraba en tratamiento con antihistamínicos. Además, tenía una descamación abundante y generalizada de la piel y marcado decaimiento. Al examinarlo se encontró un cachorro en mal estado general, enflaquecido y con signos de enfermedad generalizada de piel y linfoadenopatías, signos vitales normales, afebril y un abdomen prominente a la palpación. El pelaje era hirsuto y tenía excoriaciones
Facultad de Medicina, Universidad Andrés Bello (JLG). Hospital Veterinario Puente Alto (JL).

Complejo Asistencial Dr. Sótero del Río.

Subdirección Médica del Niño, Unidad de Infectología Pediátrica (IB, AP).

Pontificia Universidad Católica de Chile.

Laboratorio de Microbiología (MC, $\mathrm{MC}$.

Los autores declaran no tener conflictos de interés.

No hubo fuente de financiamiento.

Recibido: 6 de agosto de 2013 Aceptado: 23 de diciembre de 2013

Correspondencia a: Anamaría Peña D. anapena@med.puc.cl 
Figura 1. Descamación y alopecia de la cara y cabeza del cachorro.

Figura 2. Descamación y alopecia del cuerpo del cachorro. en todo el cuerpo, extensas zonas de alopecia, abundantes escamas y lesiones papulares pruriginosas con costras (Figuras 1 y 2).

Se le realizó un examen microscópico directo de raspado de piel y pelo que evidenció la presencia de abundantes huevos y formas juveniles y adultas de ácaros de la especie S. scabiei (Figura 3).

Se le indicó tratamiento acaricida con ivermectina inyectable semanal administrándose la primera dosis durante la consulta y baños con un producto acaricida. Durante la consulta, el propietario de la mascota refirió que él y todos los integrantes de su grupo familiar presentaban lesiones pruriginosas persistentes. Ante la sospecha de una transmisión zoonótica fueron derivados al Hospital Dr. Sótero del Río para su evaluación. En la Unidad de Infectología se efectuó una anamnesis y examen físico al padre, la madre y las cuatro hijas de cinco meses y de cuatro, siete y 12 años de edad. La madre refirió que una semana después de haber adoptado al perro, notó la aparición de pápulas costrosas, muy pruriginosas en las piernas,
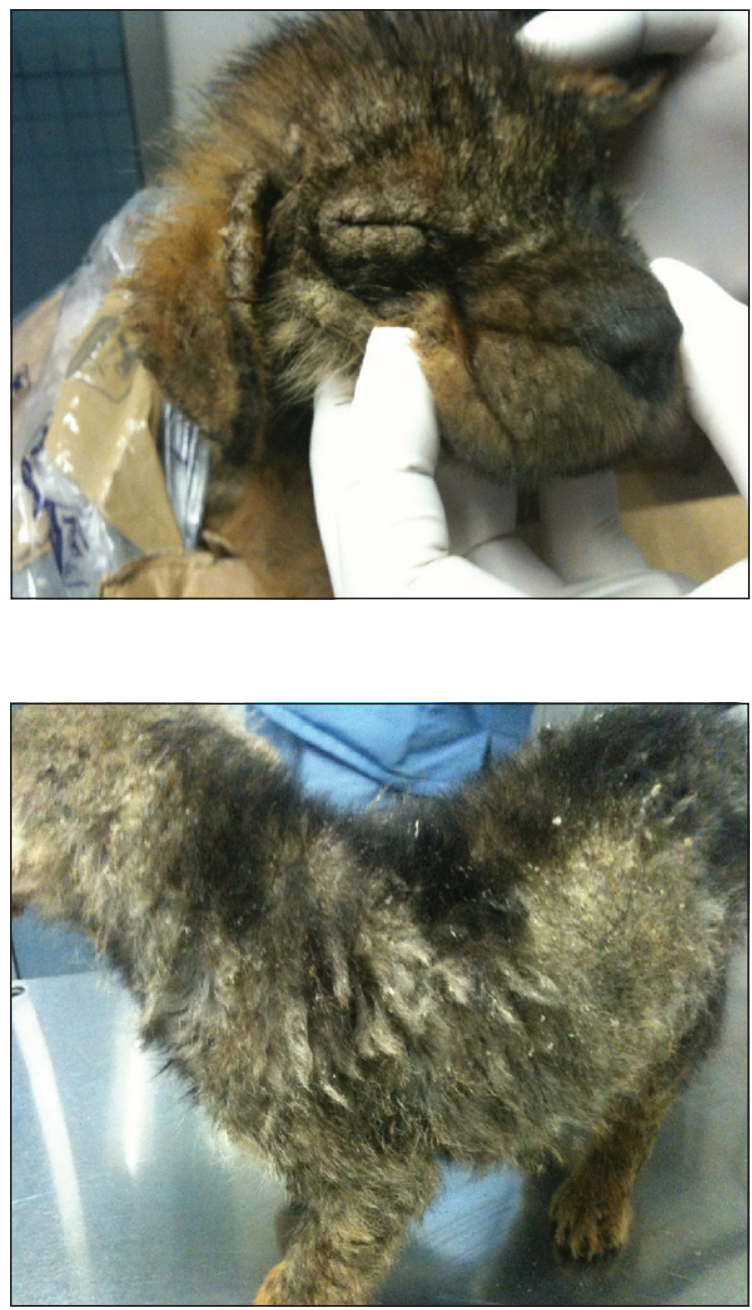

que luego se extendieron hacia el resto del cuerpo. A los pocos días el padre comenzó con la misma sintomatología y evolucionó con una dermatitis costrosa y celulitis de la pierna derecha. Finalmente, transcurridas dos semanas desde el ingreso del perro al hogar, aparecieron lesiones similares y generalizadas en todas las hijas e incluso en la abuela, quien visitaba el hogar para cuidar a las niñas. En el examen físico de ambos padres y las hijas se detectaron las lesiones descritas. (Figuras 4, 5, 6 y 7).

Se efectuó un raspado de piel para ácarotest (humano y canino) que fueron analizadas en el Laboratorio de Microbiología de la Pontificia Universidad Católica (PUC), con confirmación de resultados e informe entomológico efectuado en el Instituto de Salud Pública (ISP) y por un veterinario experto. Se realizó un hemograma a cada integrante del grupo familiar.

Se trató a la familia, incluso a la abuela, con dos pulsos de permetrina al 5\%, a excepción de la lactante y la madre que estaba dando lactancia, quienes recibieron dos pulsos de vaselina azufrada al 6\%. En ambos casos se indicó aplicar el producto tres días consecutivos, descansar cuatro días y repetirlo otros tres días. El padre y la abuela fueron tratados con dos pulsos de ivermectina oral por persistencia de las lesiones. El hemograma no demostró eosinofilia en ninguno de ellos.

Se detectó la presencia de $S$. scabiei en el ácarotest realizado a la lactante (Laboratorio de Microbiología de la PUC), con confirmación entomológica en el ISP, con lo cual se demostró la transmisión zoonótica de $S$. scabiei (Figura 8).

Posteriormente los propietarios comunicaron que el perro falleció algunas semanas después de comenzar el tratamiento.

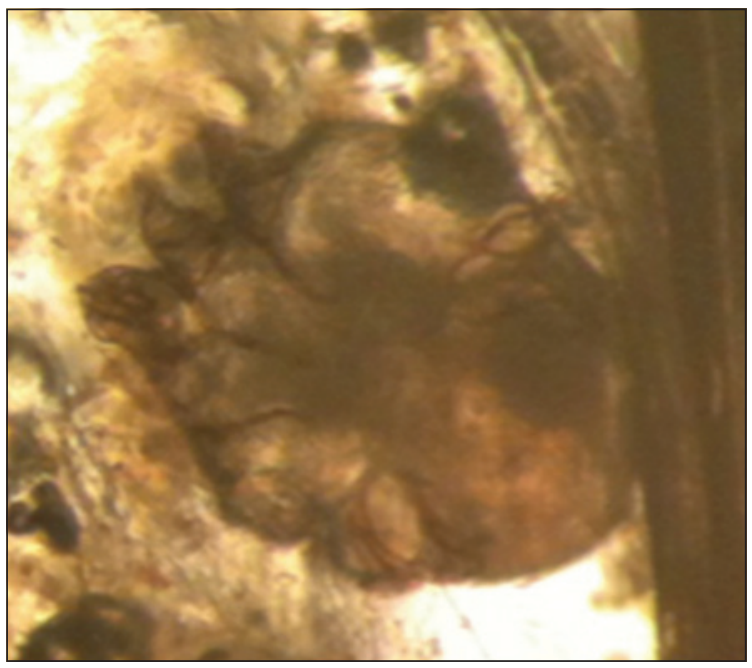

Figura 3. Ejemplar adulto de Sarcoptes scabiei en raspado de la piel del cachorro. 


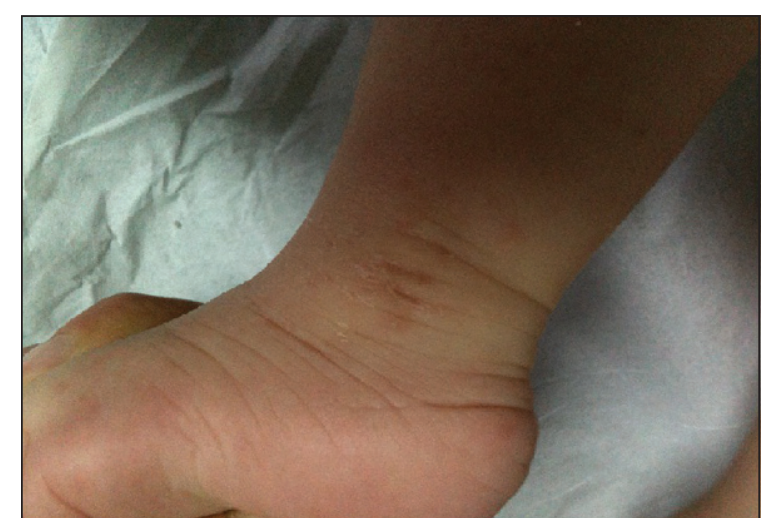

Figura 4. Lesiones papulares y costrosas en la piel de zona maleolar de la lactante.

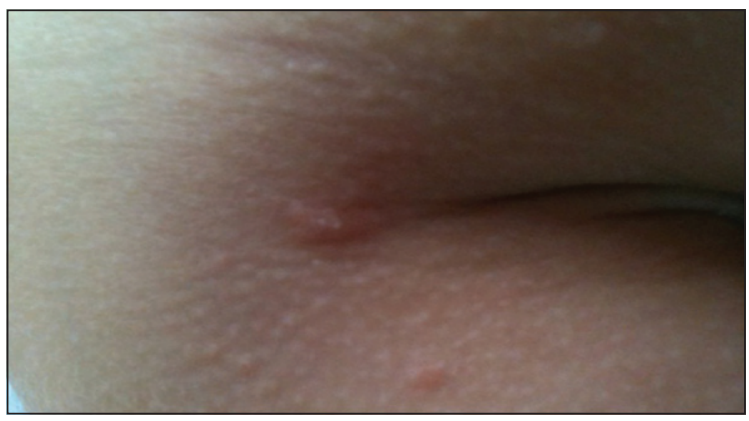

Figura 6. Lesiones papulares pruriginosas y escoriaciones en zona torácica de la niña de 7 años.

\section{Discusión}

Los ácaros son ectoparásitos cuyas diferentes especies infestan a animales y en algunos casos, causan enfermedad en hospederos humanos ${ }^{3,4,10}$. Varios de estos ácaros tienen importancia médica, especialmente en medicina veterinaria, como una de las patologías que más comúnmente afecta a los perros ${ }^{4,10,11}$. La sarna canina puede ser causada por una gran cantidad de ácaros, entre los que destacan Demodex canis, Sarcoptes scabiei var canis y Cheyletiella yasguri ${ }^{3}$.

La sarna sarcóptica, a pesar de ser la zoonosis más prevalente e importante por su amplia distribución, es una entidad sub-diagnosticada en el ser humano. Esto se debe a la falta de conocimiento entre los médicos, en contraste a lo que ocurre en medicina veterinaria, en que se le otorga un rol importante en el diagnóstico con una alta sospecha clínica.

Los ácaros se caracterizan por ser pequeños (miden entre 0,2 y $0,4 \mathrm{~mm}$ ), tienen tres o cuatro pares de patas según su estadio evolutivo (larva, ninfa y adulto) y, a diferencia de los arácnidos, poseen gnatosoma y no tienen división entre el abdomen y el cefalotórax ${ }^{10}$. El ácaro adulto de $S$.

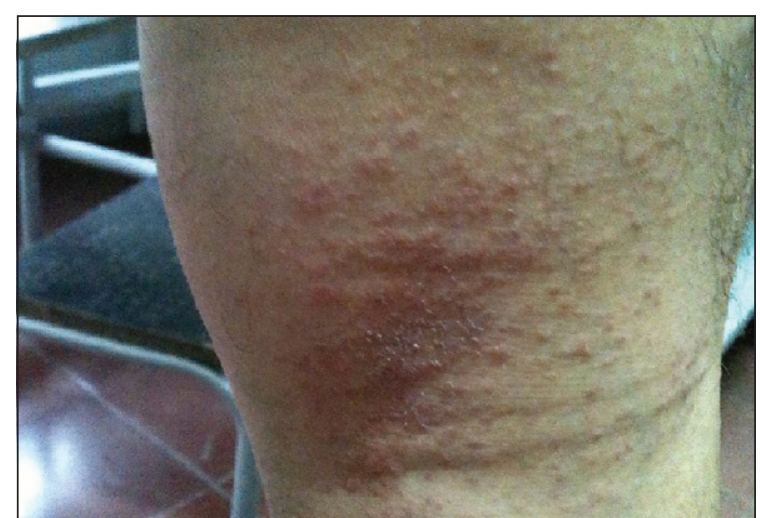

Figura 5. Lesiones papulares, costrosas y escoriaciones en el hueco poplíteo del padre.

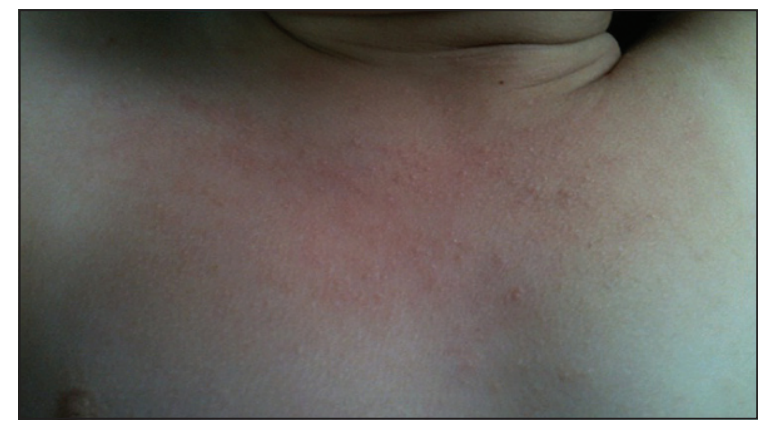

Figura 7. Lesiones papulares, costrosas y escoriaciones en el cuello de la lactante.

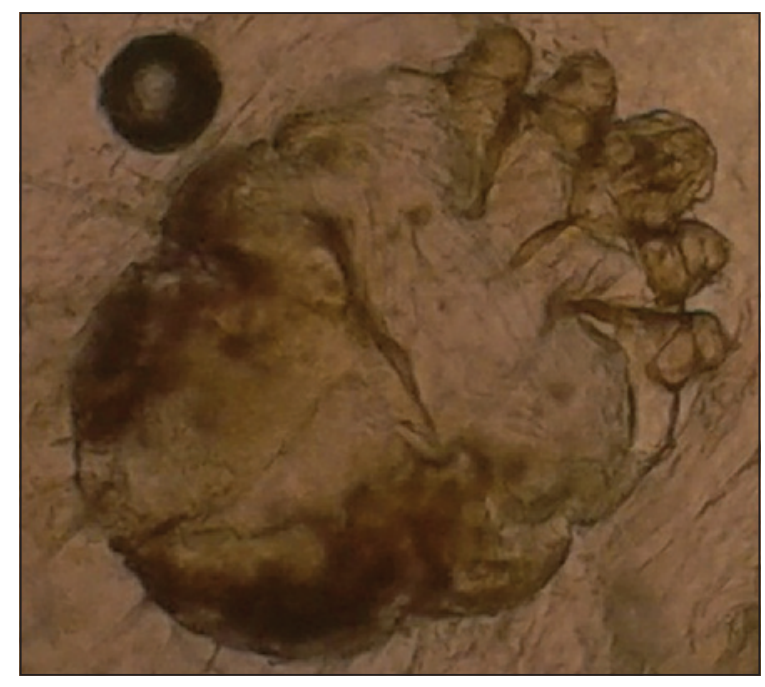

Figura 8. Ejemplar adulto de Sarcoptes scabiei en el raspado de la piel de la lactante de 5 meses.

scabiei var canis es de forma ovoide y las hembras tienen casi el doble del tamaño de los machos ${ }^{10}$. El ciclo de vida completo dura 17 a 21 días y se lleva a cabo sobre la piel del perro ${ }^{10}$. En el humano, la hembra es fecundada en la 
superficie de la piel o en la entrada de excavaciones que estas mismas hacen para depositar sus huevos en el estrato córneo, donde posteriormente mueren ${ }^{12}$.

Entre los perros la sarna sarcóptica se transmite por contacto directo y puede ser asintomática u ocasionar lesiones que habitualmente comprometen la cabeza, orejas, codos, piernas y abdomen ${ }^{13}$. Las lesiones suelen ser pápulas intensamente pruriginosas, costrosas, con excoriaciones, zonas de alopecia, inflamación e infección bacteriana secundaria, que puede causar la muerte del cachorro $^{10,12,14}$. Los perros infestados pueden transmitir el ácaro a los humanos, cuando se produce cruce entre especies; no obstante el ácaro del perro habitualmente falla en reproducirse y la infección se resuelve en forma espontánea ${ }^{4-15}$.

La sintomatología en humanos producida por la variedad canis es generalmente autolimitada, de pocas semanas de duración. La clínica es muy variada, presentándose en ocasiones de manera asintomática (portadores) o poco sintomática con lesiones en zonas de contacto con el animal infestado, generalmente localizadas en antebrazos, muslos, tórax, cuello y abdomen ${ }^{12,14}$. En los niños las zonas comprometidas son similares a las afectadas por la variedad hominis (palmas, regiones interdigitales, cabeza y cuello $)^{14,16}$. En personas inmunocompetentes y con buenos hábitos higiénicos, produce una dermatitis que aparece a las 24-96 h en las áreas de contacto, con escasas lesiones, habitualmente pápulas eritematosas, intensamente pruriginosas, sin localización típica ${ }^{10,12}$. Se describe que el prurito se intensifica en la noche y en general, aparece en forma concomitante en al menos otro integrante de la familia ${ }^{16}$. En ocasiones, puede agregarse una reacción alérgica local, secundaria a la saliva del ácaro que se deposita durante la alimentación. Debido a esto último, los principales diagnósticos diferenciales son la dermatitis alérgica, atópica y picaduras de insecto ${ }^{10,14,17,18}$. No obstante, se han descrito casos atípicos en los que la sintomatología se ha prolongado por varios meses, haciéndose persistente, pese al tratamiento adecuado. También existen reportes de brotes familiares, debido a su alta contagiosidad, con grados variables de intensidad, siendo a veces necesario agregar al tratamiento sintomático habitual, nuevos acaricidas, especialmente en pacientes con lesiones persistentes y sintomatología florida $^{10,14,19}$.

Para el diagnóstico de sarna sarcóptica es fundamental que el paciente tenga signos clínicos compatibles e historia de contacto con un animal, habitualmente con lesiones, recogido de la calle y sin control veterinario. Los exámenes de laboratorio, en general, tienen un bajo rendimiento (aproximadamente $33 \%$ ) para las variedades humanas e incluso menor para las variedades zoonóticas, debido a la baja carga parasitaria ${ }^{14}$. El examen más frecuentemente realizado es el raspado cutáneo o ácarotest, que consiste en la observación directa, bajo microscopio de una muestra de raspado de lesiones cutáneas, con la visualización del ácaro, huevos o restos de ellos, para establecer el diagnóstico por su morfología ${ }^{14,20}$. La aplicación de hidróxido de potasio $(\mathrm{KOH})$ al $20-30 \%$ sobre la muestra facilita la visualización de huevos y ejemplares de ácaros ya que disuelve las escamas de piel. En perros el diagnóstico también se realiza mediante el raspado de piel sobre la cual se aplica previamente aceite mineral el cual se distribuye uniformemente y se raspa con una hoja de bisturí 10 a 15 veces hasta que el sitio quede a punto hemorrágico. En las muestras de perros habitualmente no se visualizan los ácaros, sólo huevos grandes ovalados o pequeños restos fecales que se observan de color café. Debido a que el rendimiento alcanza sólo $25 \%{ }^{14}$, se recomienda que la muestra sea tomada desde lesiones recientes no excoriadas y de ciertas zonas como orejas, codos, tarsos, abdomen y cara ventral de tórax. Para lograr la visualización del agente se requieren múltiples muestras, tanto de personas como de perros. Otros métodos incluyen el microscopio de epifluorescencia con o sin dermatoscopio, diagnóstico serológico basado en IgE circulante o pruebas utilizando alérgenos cutáneos, aunque éstos no se encuentran disponibles en todos los laboratorios $^{14,16}$.

El tratamiento de los animales infestados con $S$. scabiei var canis se realiza con acaricidas tópicos y, en casos seleccionados, con ivermectina inyectable ${ }^{10}$. En el ser humano, el tratamiento es generalmente sintomático, con uso de antihistamínicos y corticosteroides locales; sin embargo, en los casos zoonóticos no autolimitados se recomienda el tratamiento con acaricidas ${ }^{9,14,19}$. Los tratamientos pueden ser tópicos o sistémicos. Ambos requieren de múltiples administraciones, usualmente semanales, hasta que se logre la remisión del cuadro ${ }^{13}$. Escasos estudios controlados han sido realizados para evaluar la efectividad de productos tópicos disponibles comercialmente y la selección del fármaco a menudo se basa en la preferencias del médico, disponibilidad local, edad del paciente, vía de administración y costo. Entre los acaricidas tópicos disponibles están la permetrina $5 \%$, deltametrina $0,02 \%$, lindano $1 \%$, vaselina azufrada 6-10\%, crotamitón 10\%, y en casos refractarios se recomienda el uso de ivermectina oral en dosis de $200 \mu \mathrm{g} /$ $\mathrm{kg}$ por una vez, repitiéndose a las dos semanas ${ }^{11,20,21}$. Es fundamental tratar a todos los miembros de la familia, aunque estén asintomáticos y a todos los contactos, para cortar la cadena de transmisión. 
Los escabicidas tópicos tienen efectos neurotóxicos en ácaros y estadios evolutivos más jóvenes. Estudios recientes sugieren que permetrina tópica sería la más efectiva; sin embargo, está contraindicada en el embarazo, lactancia y en recién nacidos ${ }^{20,21}$. Lindano al $1 \%$ es cancerígeno en ratas, mutagénico en bacterias y neurotóxico, y es un compuesto órganoclorado muy difícil de eliminar en la naturaleza por lo que aún, siendo aprobado por la FDA para este uso, se sugiere dejarlo como terapia de segunda línea ${ }^{22}$. Ivermectina es el único tratamiento vía oral, utilizado ampliamente en el tratamiento de strongiloidiasis y otras parasitosis, pero en su uso como anti-sárnico humano, la FDA la indica como fármaco de segunda línea, tal vez debido a su vinculación a casos de muerte súbita, por lo que no está aprobada en varios países. Puede ser utilizada a partir de 2 años de edad y sobre $15 \mathrm{~kg}$, requiriéndose de una segunda dosis debido a su falta de acción ovicida ${ }^{23-25}$. Dentro de los efectos adversos producidos por ivermectina se describe exacerbación del prurito, edema, cefalea, rash, fiebre, calofrìos, artralgias, anorexia y eosinofilia, probablemente en relación a la muerte de los parásitos al recibir el tratamiento. Existen consideraciones respecto al uso en pediatría y durante el embarazo, por la penetrancia de este fármaco a través de la barrera hemato encefálica inmadura ${ }^{26,27}$.

Entre las medidas preventivas se incluye el cuidado responsable de mascotas, el aislamiento de éstas mientras dure su enfermedad, un alto índice de sospecha frente a signos clínicos compatibles, tratamiento con acaricidas y educación para prevenir nuevas infestaciones.

Es importante tener en cuenta que la población de perros abandonados es la principal fuente de permanencia de la enfermedad. Si bien no existen datos sobre la prevalencia de la enfermedad en perros callejeros, en Chile la sarna es la cuarta causa de consulta por enfermedades infecciosas en perros, según lo establecido por el sistema de vigilancia epidemiológico veterinario ${ }^{8,9}$.

Con las técnicas habituales aplicadas en las muestras de piel del cachorro y los humanos no fue posible efectuar una diferenciación de las variedades de $S$. scabiei clásicamente descritas (canis versus hominis). lo que no permitió descartar con certeza que la infección en el grupo familiar fuese causada por S. scabiei var hominis, sin embargo, el antecedente epidemiológico apoya fuertemente la hipótesis que el agente haya sido S. scabiei var canis.La morfología de ambas variedades es muy similar y difícilmente es posible diferenciar las distintas variedades descritas, aun con técnicas sofisticadas de análisis de ADN ribosomal. Algunos autores postulan que existiría una sola especie pero variable dado que existirían diferencias fisiológicas entre los ácaros obtenidos de diferentes hospederos ${ }^{2}$. Se cree que la variablidad morfológica y ecológica de S. scabiei es resultado no tanto de una adaptación a diferentes especies animales sino más bien de cruzas continuas entre diferentes variedades. Este fenómeno ha actuado contra el proceso de especiación, incrementando su variabilidad genética y permitiendo que el género Sarcoptes pueda infectar nuevas especies lo que explicaría la infección zoonótica ${ }^{2}$.

\section{Conclusiones}

La sarna sarcóptica es una infección muy prevalente en mascotas caninas y su alta contagiosidad le confiere un elevado potencial zoonótico. Debido a que en el humano su presentación clínica difiere del patrón característico de la sarna humana, generalmente se confunde con dermatitis alérgica, atópica o incluso picaduras de insecto y el tratamiento específico se retrasa. Por lo tanto, debe considerarse en el diagnóstico diferencial de la alergia persistente e intensamente pruriginosa que no cede con el tratamiento sintomático habitual, en pacientes que han tenido contacto prolongado y estrecho con animales en malas condiciones de salud. El tratamiento debe considerar el uso de acaricidas cuando las lesiones son persistentes y el control de la enfermedad requiere el adecuado tratamiento de la mascota. Una medida preventiva primordial es efectuar un examen veterinario previo a la adopción e introducción de mascotas abandonadas al hogar.

\section{Resumen}

La sarna producida por el género Sarcoptes scabiei var canis, infección prevalente en perros y de alto potencial zoonótico, afecta a animales abandonados, desnutridos y hacinados y causa alopecia y una dermatitis costrosa intensamente pruriginosa. En el ser humano produce una dermatitis pruriginosa generalmente autolimitada, pero se describen casos persistentes. Se reporta un brote de sarna sarcóptica en un grupo familiar (siete personas, incluidas una lactante y su madre) cuya fuente de infección fue su mascota canina recogida de la calle. El diagnóstico fue confirmado por visualización en el ácarotest de ácaros y huevos en el perro y ácaros de S. scabiei en la lactante. La sarna sarcóptica debe sospecharse en casos de dermatitis alérgica en personas con contacto con perros. El tratamiento en el humano, habitualmente sintomático, puede necesitar acaricidas si el cuadro persiste. El control de la enfermedad requiere el adecuado tratamiento de la mascota. 


\section{Referencias bibliográficas}

1.- Heukelbach J, Feldmeier H. Scabies. Lancet 2006; 367: 1767-74.

2.- Aydıngöz I E, Mansur A T. Canine scabies in humans: a case report and review of the literature. Dermatology 2011; 223: 104-6.

3.- Feather L, Gough K, Flynn R J, Elsheikha H M. A retrospective investigation into risk factors of sarcoptic mange in dogs. Parasitol Res 2010; 107: 279-83.

4.- Nuttall T. Introduction. Veterinary advice on skin disorders in dogs. $1^{\text {st }}$ edition, Ringpress, Dorking; 2004, p. 4-6.

5.- Berck W, Pfister K. Mites as a cause of zoonoses in human beings. Wien Klin Wochenschr 2006; 118 (Suppl 3): 27-32.

6.- Demis J D. Injurious effects in man induced by animals, Scabies (itch mites). Demis J D, Clinical Dermatology. 25 $\mathrm{Ed}$, Philadelphia, Lippincot Co 1994; Unit 18-32 (3): 1-6.

7.- Currie B J, McCarthy J S. Permethrin and ivermectin for scabies. N Engl J Med 2010; 362 : 717-25.

8.- López J, Abarca K, Cerda J, Valenzuela B, Lorca L, Olea A, et al. Surveillance system for infectious diseases of pets, Santiago, Chile. Emerg Infect Dis 2009; 15: 1674-6.

9.- Abarca K, Jofré L, López J, Lorca L. Pauta técnica de vigilancia de enfermedades transmisibles en pequeños animales de compañía. Disponible en http://www.sochinf. cl/sitio/templates/sochinf2008/documentos/ pautaTecnica_animales.pdf. (Accedido: $10 \mathrm{de}$ junio de 2013).
10.- Jofré L, Noemí I, Neira P, Saavedra T, Díaz C. Acarosis y zoonosis relacionadas. Rev Chilena Infectol 2009; 26: 248-57.

11.- Krauss H, Weber A, Apple M, Enders B. Isenberg H D, Schiefer H G. Chapter 4: Parasitic zoonoses caused by mites. Zoonoses infectious disease transmissible from animals to humans. $3^{\text {th }}$ edition, ASM Press, Washington; 2003, p. 399-402.

12.- Apt W. Capítulo 78: Sarna y otras acariasis. Apt W. Parasitología Humana. $1^{\mathrm{a}}$ edición, Ed. McGraw-Hill Interamericana Editores S. A. de C.V.; 2013, p. 638.

13.- Scoot D, Miller M, Griffin C. Parasitic skin diseases. Muller and Kirk's Small Animal Dermatology. $6^{\text {th }}$ edition. W. B. Saunders, Philadelphia, P A, USA; 2000, p. 476-83.

14.- Saavedra T, Díaz C, Leiva A, Zapata S. Sarna sarcóptica transmitida a humano. Rev Chil Dermatol 2007; 23: 302-4.

15.- Rabinowitz P M, Gordon Z, Odofin L. Pet-related infections. Am Fam Physician 2007; 76: 1314-22.

16.- Monsel G, Chosidow O. Management of scabies. Skin Therapy Lett 2012; 17: 1-4.

17.- Cheng T C. Capítulo: Ácaros. Cheng T C. Parasitología General. Segunda edición 1980. Editorial AC. Madrid, España. P. 751-77.

18.- Diamantis S A, Morrell D S, Burkhart C N. Pediatric infestations. Pediatr Ann 2009; 38: 326-32.

19.- Larsson M H. Evidências epidemiológicas da ocorrência de escabiose, em humanos, causada pelo Sarcoptes scabiei (De Geer, 1778) var canis (Bourguignon, 1853). Rev Saúde Públ S
Paulo 1978; 12: 333-9.

20.- Guía Clínica de Sarna y Pediculosis 2007. MINSAL. Disponible en: http://www.redsalud. gov.cl/portal/url/item/5d26b93e3caca628e040 01011e0104c7.pdf. (Accedido: 10 de junio de 2013)

21.- Strong M, Johnstone P. Interventions for treating scabies. Cochrane Database Syst Rev. 2007(3): CD000320.

22.- FDA AERS database search, January 2003. Lindane Lotion Medguide. En http://www. fda.gov/downloads/drugs/../UCM.133687.pdf (Accedido: 21 de diciembre de 2013)

23.- Chosidow O. Clinical practice. Scabies. N Engl J Med 2006; 354: 1718-27.

24.- Currie B J, McCarthy J S. Permethrin and ivermectin for scabies. N Engl J Med 2010; 362: 717-25.

25.- Barkwell R, Shield S. Deaths associated with ivermectin treatment of scabies. Lancet 1997; 349: 1144-5.

26.- Jaramillo-Ayerbe F, Berrío-Muñoz J. Ivermectin for crusted Norwegian scabies induced by use of topical steroids. Arch Dermatol 1998; 134: 143-5.

27.- Fawcett R S. Ivermectin use in scabies. Am Fam Physician 2003; 68: 1089-92.

28.- Arlian L G. Biology, host relations, and epidemiology of Sarcoptes scabiei. Annu Rev Entomol 1989; 34: 139-61.

29.- Berilli F, D’Amelio S, Rossi L. Ribosomal and mitochondrial DNA sequence variation in Sarcoptes mites from different hosts and geographical regions. Parasitol Res 2002; 88: $772-7$. 